\title{
Searches for Dark Matter via Mono-Higgs signatures with the CMS experiment
}

\author{
Giorgia Miniello* on behalf of the CMS Collaboration \\ Università and INFN Bari \\ E-mail: giorgia.miniello@ba.infn.it
}

The Higgs boson discovery in 2012 represented not only one of the most important event occurred in the latest history of Physics, but also opened a new scenario in the detection of possible Dark Matter candidates that could be produced at colliders. In this context, the Higgs boson can be used as a probe for Dark Matter discovery. The study of the Dark Matter, whose cosmological evidence is definitely established, is one of the most promising and exciting frontier of the Physics research. The signature of the so-called Mono-Higgs analyses are typically characterized by a Higgs boson and missing transverse energy in the final state. Since the production of the Higgs via the initial state radiation is highly suppressed due to the small coupling with the quarks, an evidence of Mono-Higgs signal would probe directly the structure of the effective DM-SM coupling.

A complete overview of all the latest results from the Mono-Higgs analyses performed with the CMS experiment at the LHC will be provided using data collected during 2015 and 2016 at a luminosity of $2.3 \mathrm{fb}^{-1}$ and $35.9 \mathrm{fb}^{-1}$ respectively.

The European Physical Society Conference on High Energy Physics

5-12 July, 2017

Venice

* Speaker. 


\section{The Mono-Higgs Analysis}

The Higgs boson $(\mathrm{H})$ discovery in 2012 opened a new interesting window for searching Dark Matter (DM) at the LHC via the Mono-Higgs signature. So far, no evidence of dark matter candidates have been found at colliders even though, starting from the assumption that DM is indeed associated with the scale of electroweak symmetry breaking [1,2], it can be asserted that colliders are a natural place to search for Higgs-boson-related signatures. An exploration of possible DM-Higgs boson couplings have been already performed studying invisible Higgs decays but all the searches at LHC based on this sector led to null results [3]. The expected signature of DM at colliders is typically characterized by a large value of missing transverse energy, denoted $E_{T}^{\text {miss }}$ or MET, due to DM candidates which do not interact with the detector. Mono-Higgs analyses search for final states with Higgs boson products plus a large value of missing transverse energy. They are characterized by a highly suppressed ISR due to the small coupling of the Higgs to quarks. Therefore, one Higgs boson is preferentially emitted from the new physics vertex. This would directly probe the effective DM-SM coupling and so it can be said that the Higgs is used as a probe.

\section{The Signal Benchmark Models}

For the Mono-Higgs analyses here presented two benchmark simplified models have been considered. The first one is the $Z^{\prime} 2 H D M$, where a $Z^{\prime}$ is produced as an on-shell resonance decaying into a Higgs and a pseudoscalar $A_{0}$, with $A_{0}$ decaying into a pair of particle-antiparticle DM candidates. The other model used is the the $Z^{\prime}$ Baryonic, where a vector mediator $Z_{B}^{\prime}$ decays into $\chi \bar{\chi}$ after radiating a Higgs [4]. The main parameters of the two models considered are summarised in Table 1.

\begin{tabular}{|c|c|c|c|}
\hline Model & Parameter & Description & Value \\
\hline$Z^{\prime}$ 2HDM & $m_{A_{0}}$ & Mass of $A_{0}$ & $300 \mathrm{GeV}$ \\
& $g_{Z^{\prime}}$ & $Z^{\prime}$ ' coupling strenght & 0.8 \\
& $m_{\chi}$ & Mass of DM candidate & $100 \mathrm{GeV}$ \\
& $m_{Z^{\prime}}$ & Mass of $Z^{\prime}$ & $600-2500 \mathrm{GeV}$ \\
& $\tan \beta$ & Ratio between the two vevs of Higgs from $Z^{\prime} 2 H D M$ & 1 \\
\hline$Z^{\prime}$ Baryonic & $g_{Z_{B}^{\prime}}$ & $D M-Z_{B}^{\prime}$ coupling & 1 \\
& $g_{q}$ & mediator-quark coupling & 0.25 \\
& $g_{h Z^{\prime} Z_{B}^{\prime}} / m_{Z^{\prime}}$ & coupling between $Z_{B}^{\prime}$ and the SM-like Higgs & 1 \\
& $\sin \theta$ & mixing angle between baryonic Higgs and the SM-like Higgs & 0.3 \\
& $m_{Z_{B}}$ & mass of $Z_{B}^{\prime}$ & $10-1000 \mathrm{GeV}$ \\
& $m_{\chi}$ & Mass of DM candidate & $1-1000 \mathrm{GeV}$ \\
\hline
\end{tabular}

Table 1: Main parameters of $Z^{\prime} 2 H D M$ and $Z^{\prime}$ Baryonic models. In the Table vevs stands for vacuum expectation values.

\section{Dark Matter produced in association with a Higgs boson decaying to Two Photons}

The Mono-Higgs $\gamma \gamma$ analysis used 2016 data collected during p-p collisions at $\sqrt{s}=13 \mathrm{TeV}$ corresponding to an integrated luminosity $\mathscr{L}=35.9 \mathrm{fb}^{-1}$. Results are interpreted using both 
$Z^{\prime} 2 H D M$ and $Z^{\prime}$ Baryonic models. The analysis strategy includes many pre-selection requirements that must be applied to photon candidates. Among others, good photon candidates must be chosen in the fiducial ECAL range $(\eta<1.4442$ or $1.566<\eta<2.5)$, the transverse momentum of the photon $p_{T}^{\gamma}$ must be chosen so that $p_{T}^{\gamma}>30 \mathrm{GeV}$ for the leading one $\left(p_{T}^{\gamma}>20 \mathrm{GeV}\right.$ for the subleading), the mass of the diphoton must be $m_{\gamma \gamma}>95 \mathrm{GeV}$ and the ratio between the mass of the transverse momentum and the mass of the diphoton must be $p_{T}^{\gamma} / m_{\gamma \gamma}>1 / 3(1 / 4)$ for the leading (subleading) photon. The cut on transverse momentum $p_{T}$ is chosen so that the sculpting of shape is avoided. Different photon identification criteria, relied on photon related variable (e.g. the ratio of hadronic to electromagnetic energy $H / E$ ) are applied to photons in the barrel and in the endcaps.

The selection criteria applied on the preselected candidates consist in choosing photon pairs to form a diphoton object. The analysis looks for an excess on the diphoton invariant mass spectrum plus a large missing transverse momentum. The missing transverse momentum of the selected events and the diphoton invariant mass are the two main variables used.

The main irreducible background source for this analysis is the associated production samples of the Higgs with a vector boson, $\mathrm{VH}$ (where $\mathrm{V}=\mathrm{Z}, \mathrm{W}$ ), with $\mathrm{Z}$ decaying into two neutrinos and $\mathrm{W}$ decaying into a lepton and a neutrino.

The reducible backgrounds can be classified into resonant ( $g g F, V B F, t t H)$ and non-resonant sources (QCD, EWK processes).

Figure 1 shows on the left the 95\% Confidence Level (CL) upper limits on the cross section for the $Z^{\prime} 2 H D M$ scenario as a function of $m_{Z}^{\prime}$ for $m_{A_{0}}=300 \mathrm{GeV}$ (at which signal cross section has the maximum value). 95\% CL upper limits on the cross section for the $Z^{\prime}$ Baryonic model as a function of $m_{Z_{B}^{\prime}}$ for $m_{\chi}=1 \mathrm{GeV}$ is shown on the right. For more details on the analysis see [5].
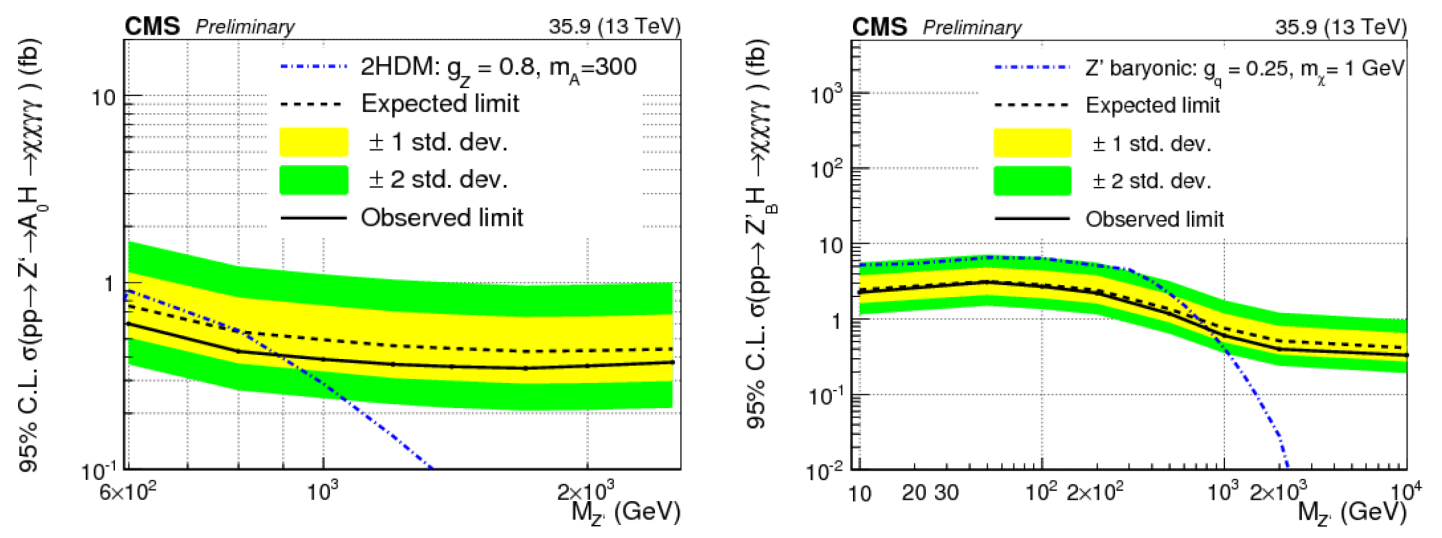

Figure 1: The upper limits on cross section for the $Z^{\prime} 2 H D M$ scenario $(l e f t)$ and for the Z? Baryonic model (left) for Mono-Higgs $\gamma \gamma$

\section{Dark Matter produced in association with a Higgs boson decaying into a pair of bottom quarks}

The Mono-Higgs analysis with $\mathrm{H}$ decaying in $b \bar{b}$ used 2015 data collected during p-p collisions at $\sqrt{s}=13 \mathrm{TeV}$ corresponding to a luminosity $\mathscr{L}=2.3 \mathrm{fb}^{-1}$. The results are interpreted using 
the $Z^{\prime} 2 H D M$ approach. This study uses the resolved regime for lower values of $m_{Z^{\prime}}$ (from 600 to $1000 \mathrm{GeV}$ ) in which the Higgs boson gives rise to two separate b-jets with a minimum angular distance between the decay products of $H(b \bar{b}) \Delta R=0.4$ and the boosted one for higher values of $m_{Z^{\prime}}(>1000 \mathrm{GeV}$ ), in which the Higgs boson is reconstructed by one single jet with a jet radius $\Delta R$ $=0.8$. This analysis search for final events with large $E_{T}^{\text {miss }}$, trigger requirements both on $E_{T}^{\text {miss }}$ and $H_{T}^{\text {miss } 1}$ and cut on multijets. The main irreducible background source for this analysis is $\mathrm{Z}+j e t s$, with $\mathrm{Z}$ decaing in two neutrinos, while the reducible backgroud sources come from multijet events, $\mathrm{W}+$ jets plus top quark production, $\mathrm{Z}+$ jets, diboson production and VH. Figure 2 shows the $95 \%$ CL upper limits on the cross section for the $Z^{\prime} 2 \mathrm{HDM}$ scenario as a function of $m_{Z}^{\prime}$ for $m_{A 0}=300$ $\mathrm{GeV}$. For $m_{Z}^{\prime}=600,800,1000 \mathrm{GeV}$ resolved regime has been used while for the other higher mass points the boosted approach has been chosen. For more details on the signal event selection for both the regimes of this analysis see [6].

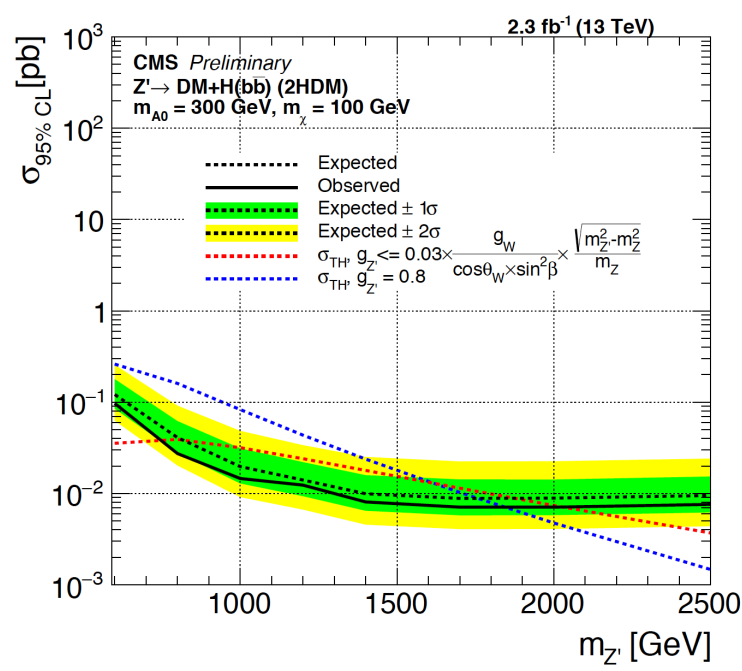

Figure 2: Expected and observed 95\% CL limits on dark matter production cross section for Mono-Higgs $b \bar{b}$ analysis. For $m_{Z}^{\prime}=600,800,1000 \mathrm{GeV}$ resolved analysis has been used while the other higher mass points have been chosen for the boosted regime.

\section{Conclusions}

No evidence for DM production has been observed from both the Mono-Higgs studies here presented . For Mono-Higgs $\gamma \gamma$ the $Z^{\prime} 2 \mathrm{HDM}$ signals with $m_{A_{0}}=300 \mathrm{GeV}$ are excluded at $95 \% \mathrm{CL}$ for $m_{Z^{\prime}}<900 \mathrm{GeV}$ while the $Z^{\prime}$ Baryonic signals with $m_{\chi}=1 \mathrm{GeV}$ are excluded at $95 \%$ CL for $m_{Z^{\prime}}$ $<800 \mathrm{GeV}$. The Mono-Higgs $b \bar{b}$ analysis results for $Z^{\prime} 2 \mathrm{HDM}$ approach shows that the range 600 $<m_{Z^{\prime}}<1777 \mathrm{GeV}$ is expected to be excluded at $95 \% \mathrm{CL}$, while the range $600<m_{Z^{\prime}}<1863 \mathrm{GeV}$ is excluded from the observed data.

\footnotetext{
${ }^{1} H_{T}^{m i s s}$ is the vectorial sum of the transverse momenta of all jets with $p_{T}>20 \mathrm{GeV}$
} 


\section{References}

[1] G. Jungman, M. Kamionkowski, K. Griest, Supersymmetric Dark Matter, 10.1016/0370-1573(95)00058-5, 1995

[2] G. Bertone, D. Hooper, J. Silk, Particle Dark Matter: Evidence, Candidates and Constraints, 10.1016/j.physrep.2004.08.031, 2004

[3] L. Carpenter et al, Mono-Higgs: a new collider probe of dark matter, 10.1103/PhysRevD.89.075017, 2013

[4] Abercrombie et al., Dark Matter Benchmark Models for Early LHC Run-2 Searches: Report of the ATLAS/CMS Dark Matter Forum, arXiv:1507.00966v1 [hep-ex], 2015

[5] The CMS Collaboration, earch for Dark Matter Produced in Association with a Higgs Boson Decaying to Two Photons, CMS PAS EXO-16-054, 2016

[6] The CMS Collaboration, Search for dark matter in association with a Higgs boson decaying into a pair of bottom quarks at $\sqrt{s}=13$ TeV with the CMS detector CMS PAS EXO-16-012, 2016 